\title{
Cardiac sympathetic burden reflects Parkinson disease burden, regardless of high or low orthostatic blood pressure changes
}

\author{
Sang-Won Yoo ${ }^{1}$, Joong-Seok Kim (iD ${ }^{1 凶}$, Yoon-Sang Oh${ }^{1}$, Dong-Woo Ryu' ${ }^{1}$, Seunggyun Ha ${ }^{2}$, Ji-Yeon Yoo (D) and Kwang-Soo Lee ${ }^{1}{ }^{1}$
}

Reduced uptake of ${ }^{123}$-meta-iodobenzylguanidine $\left({ }^{123} \mathrm{I}-\mathrm{MIBG}\right)$ and orthostatic hypotension $(\mathrm{OH})$ are independently associated with worse clinical outcomes of Parkinson's disease (PD). However, their interactive influence on PD has not been studied. The role of ${ }^{123}$ I-MIBG myocardial uptake, as a biomarker of PD severity, was investigated, conditional on the mediating effects of OH. A total of 227 PD patients were enrolled. Their motor and nonmotor aspects were assessed with standardized tools. Global disease burden was estimated by averaging the scaled z-scores of the assessment tools. Every patient went through ${ }^{123}$ I-MIBG scan, and OH was evaluated with the head-up tilt-test. The mediating role of orthostatic blood pressure changes $(\triangle \mathrm{BP})$ on the association between cardiac sympathetic denervation and disease burden was investigated. Low heart-to-mediastinum $(\mathrm{H} / \mathrm{M})$ ratio with less than 1.78 was seen in $69.6 \%$ of the patient population, and $22.9 \%$ of patients had $\mathrm{OH}$. Low $\mathrm{H} / \mathrm{M}$ ratio was associated with $\mathrm{OH}$, and these patients had worse disease burden than subjects with normal ${ }^{123}$ I-MIBG uptake (global composite $z$-score: normal ${ }^{123}$ I-MIBG vs. abnormal ${ }^{123}$-MIBG; $-0.3 \pm 0.5$ vs. $0.1 \pm 0.7 ; p<0.001$ ). The mediation models, controlled for age and disease duration, revealed that the delayed $\mathrm{H} / \mathrm{M}$ ratio and global composite score were negatively associated, irrespective of orthostatic $\triangle \mathrm{BP}$. Adverse relationship between cardiac sympathetic denervation and disease burden was shown without any interference from orthostatic blood pressure fluctuations. This result suggested that extracranial cardiac markers might reflect disease burden, regardless of labile blood pressure influence.

npj Parkinson's Disease (2021)7:71 ; https://doi.org/10.1038/s41531-021-00217-3

\section{INTRODUCTION}

Cardiovascular dysautonomia is increasingly accepted as a prodromal 'window' in which Parkinson's disease (PD) can be detected $^{1-5}$. These findings have been reported as clinical biomarkers for predicting PD clinical outcomes and consequences $^{6-10}$.

Cardiac postganglionic sympathetic denervation, reflected more accurately in the decreased delayed heart-tomediastinum $(\mathrm{H} / \mathrm{M})$ ratio upon ${ }^{123} \mathrm{I}$-meta-iodobenzylguanidine $\left({ }^{123}\right.$ I-MIBG) myocardial scintigraphy scan ${ }^{11}$, contributes to orthostatic hypotension $(\mathrm{OH})^{4}$. Reduced myocardial uptake of ${ }^{123}$ I-MIBG indicates norepinephrine transporter dysfunction in sympathetic neurons, and may reflect physiological consequences in residual functional cardiac sympathetic axons ${ }^{7,12}$. Reduced uptake of ${ }^{123}$ I-MIBG was utilized to discriminate PD from vascular Parkinsonism, drug-induced Parkinsonism, and atypical parkinsonian syndromes (APS) ${ }^{2}$.

Some PD patients have a normal ${ }^{123}$ I-MIBG uptake analogous to APS, and this imaging phenotype was previously given the term "scans without evidence of cardiac norepinephrine deficit (SWEND)"13. PD patients with SWEND had mild Hoehn and Yahr $(\mathrm{H} \& \mathrm{Y})$ stage, short disease duration, slow progression of motor dysfunction, a lower incidence of the wearing-off phenomenon, and a lower prevalence of nonmotor symptoms ${ }^{7,10-12,14,15}$.

Most studies have investigated the association of cardiac sympathetic denervation with orthostatic hypotension and the impact on clinical outcomes separately, but the interacting effects of these two biomarkers on PD patient symptoms have seldom been explored ${ }^{4,16}$. As a pathophysiologic contributor, $\mathrm{OH}$ is a possible biomarker of pathologic burdens $s^{2,4,7,12,17}$, thus the confounding effects of $\mathrm{OH}$ need to be considered when assessing the role of ${ }^{123}$ I-MIBG myocardial scintigraphy results in PD patients.

In this study, we investigated whether a mediating role for $\mathrm{OH}$ could be disproved in the context of a significant association between cardiac sympathetic denervation and clinical disease burden. The refutation of this role would emphasize the sustained value of ${ }^{123}$ I-MIBG uptakes as a pathologic biomarker of disease severity despite its contribution to $\mathrm{OH}$.

Orthostatic hypertension (OHT) is also a type of cardiovascular dysregulation which can be anticipated in PD patients ${ }^{18,19}$. This study also aimed to document the nature of OHT in early PD patients.

\section{RESULTS}

\section{Baseline characteristics}

Baseline characteristics are summarized in Table 1. A total of 227 patients with mild PD stage were included. The mean age of the population was $69.6 \pm 9.2$ years old, and 105 (46.3\%) were female. The disease duration was $1.1 \pm 1.0$ years. Total Unified Parkinson's Disease Rating Scale (UPDRS) and H\&Y were 23.4 \pm 11.9 and 2.0 (Interquartile range, IQR, 0.0), respectively. The Mini-Mental Status Examination (MMSE) was $26.8 \pm 3.0$ and Clinical Dementia Rating (CDR) was $0.5(\mathrm{IQR}, 0.0)$. The mean uptake of early and delayed ${ }^{123} \mathrm{I}$-MIBG H/M ratio was $1.58 \pm 0.31$ and $1.55 \pm 0.37$, respectively. $69.6 \%(158 / 227)$ of patients were defined as having decreased $\mathrm{H} / \mathrm{M}$ ratio on ${ }^{123} \mathrm{I}-\mathrm{MIBG}$ scintigraphy (normal ${ }^{123} \mathrm{I}-\mathrm{MIBG}$ group vs. abnormal ${ }^{123}$ I-MIBG group: $2.04 \pm 0.16$ vs. $1.34 \pm 0.18$, respectively). Twenty patients were found to be hypertensive when in the supine position; $40 \%(8 / 20)$ and $30 \%(6 / 20)$ of patients with supine hypertension $(\mathrm{SH})$ had co-existing $\mathrm{OH}$ and $\mathrm{OHT}$, respectively.

${ }^{1}$ Department of Neurology, College of Medicine, The Catholic University of Korea, Seoul, Republic of Korea. ${ }^{2}$ Division of Nuclear Medicine, Department of Radiology, College of Medicine, The Catholic University of Korea, Seoul, Republic of Korea. ${ }^{凶}$ email: neuronet@catholic.ac.kr 
Table 1. Baseline characteristics.

\begin{tabular}{|c|c|c|c|c|}
\hline & $\mathrm{PD}(n=227)$ & Normal ${ }^{123}$ I-MIBG $(n=69)$ & Abnormal ${ }^{123}$ I-MIBG $(n=158)$ & $p$ value \\
\hline Sex, female, $n(\%)$ & $105(46.3)$ & $35(50.7)$ & $70(44.3)$ & 0.389 \\
\hline Disease duration, years, mean (SD) & $1.1(1.0)$ & $1.0(1.0)$ & $1.2(1.0)$ & 0.399 \\
\hline Diabetes mellitus, $n$ (\%) & $39(17.2)$ & $14(20.3)$ & $25(15.8)$ & 0.446 \\
\hline Non-smoker, $n(\%)$ & $222(97.8)$ & $68(98.6)$ & $154(97.5)$ & 1.000 \\
\hline UPDRS, total, mean (SD) & $23.4(11.9)$ & $20.4(11.1)$ & $24.8(12.0)$ & 0.010 \\
\hline UPDRS Part I, mean (SD) & $1.6(1.4)$ & $1.4(1.3)$ & $1.7(1.4)$ & 0.222 \\
\hline UPDRS Part II, mean (SD) & $6.1(4.1)$ & $5.3(4.0)$ & $6.4(4.1)$ & 0.074 \\
\hline UPDRS Part III, mean (SD) & $15.8(8.6)$ & $13.6(8.0)$ & $16.7(8.7)$ & 0.013 \\
\hline H\&Y stage $2, n(\%)$ & $146(64.3)$ & $40(58.0)$ & $106(67.1)$ & \\
\hline H\&Y stage $3, n(\%)$ & $25(11.0)$ & 7 (10.1) & $18(11.4)$ & \\
\hline H\&Y stage $4, n(\%)$ & $1(0.4)$ & $0(0.0)$ & $1(0.6)$ & \\
\hline MMSE, mean (SD) & $26.8(3.0)$ & $26.8(3.4)$ & $26.9(2.9)$ & 0.844 \\
\hline CDR, median (IQR) & $0.5(0.0)$ & $0.5(0.0)$ & $0.5(0.0)$ & 0.812 \\
\hline Early H/M ratio, mean (SD) & $1.58(0.31)$ & $1.96(0.18)$ & $1.41(0.18)$ & $<0.001$ \\
\hline Delayed H/M ratio, mean (SD) & $1.55(0.37)$ & $2.04(0.16)$ & $1.34(0.18)$ & $<0.001$ \\
\hline Washout rate, mean (SD) & $2.18(8.37)$ & $-4.87(6.81)$ & $5.25(7.03)$ & $<0.001$ \\
\hline Supine SBP, mean (SD) & $124.1(16.5)$ & $122.7(17.2)$ & $124.6(16.2)$ & 0.428 \\
\hline Supine DBP, mean (SD) & $71.0(9.1)$ & $70.1(9.0)$ & $71.4(9.1)$ & 0.317 \\
\hline orthostatic $\Delta \mathrm{SBP}_{\min }$, mean (SD) & $10.6(13.3)$ & $5.3(11.0)$ & $12.9(13.6)$ & $<0.001$ \\
\hline MADRS, total, median (IQR) & $4.0(7.0)$ & $3.0(5.0)$ & $4.0(7.0)$ & 0.024 \\
\hline ESS, total, median (IQR) & $2.0(3.5)$ & $2.0(3.0)$ & $3.0(3.0)$ & 0.012 \\
\hline PDSS2, total, mean (SD) & $7.4(6.8)$ & $5.3(5.2)$ & $8.3(7.2)$ & 0.001 \\
\hline RBDSQ, total, mean (SD) & $3.2(2.6)$ & $1.9(1.5)$ & $3.8(2.7)$ & $<0.001$ \\
\hline SCOPA-AUT, total, mean (SD) & $8.1(6.7)$ & $6.2(5.7)$ & $8.9(6.9)$ & 0.003 \\
\hline OHQ Part I, subtotal, median (IQR) & $0.0(9.0)$ & $0.0(4.0)$ & $0.0(10.0)$ & 0.133 \\
\hline OHQ Part II, subtotal, median (IQR) & $0.0(7.0)$ & $0.0(2.0)$ & $0.0(8.0)$ & 0.004 \\
\hline Global composite score, z, mean (SD) & $0.0(0.7)$ & $-0.3(0.5)$ & $0.1(0.7)$ & $<0.001$ \\
\hline Motor composite score, $z$, mean (SD) & $0.0(0.89)$ & $-0.2(0.9)$ & $0.1(0.9)$ & 0.016 \\
\hline Sleep composite score, $z$, mean (SD) & $0.0(0.76)$ & $-0.4(0.5)$ & $0.2(0.8)$ & $<0.001$ \\
\hline Autonomic composite score, z, mean (SD) & $0.0(0.80)$ & $-0.2(0.7)$ & $0.1(0.8)$ & 0.006 \\
\hline
\end{tabular}

The values of blood pressures are expressed in $\mathrm{mmHg}$.

Independent $t$-test or Welch's $t$-test was conducted for continuous variables and Fisher's exact test was used for categorical variables. Non-normally distributed variables were analyzed by Mann-Whitney $U$ tests.

PD Parkinson's disease, MIBG metaiodobenzylguanidine, UPDRS Unified Parkinson's Disease Rating Scale, H\&Y Hoehn and Yahr, MMSE Mini-Mental Status Examination, $C D R$ Clinical Dementia Rating, SBP systolic blood pressure, $D B P$ diastolic blood pressure, $S H$ supine hypertension, $O H$ orthostatic hypotension, $O H T$ orthostatic hypertension, H/M heart-to-mediastinum, NMSS Nonmotor Symptoms Scale, MADRS Montgomery-Asberg Depression Rating Scale, ESS Epworth Sleepiness Scale, PDSS2 Parkinson's Disease Sleep Scale-2, RBDSQ REM Sleep Behavior Disorder Screening Questionnaire, SCOPA-AUT Scale for Outcomes in Parkinson's Disease-Autonomic, $\mathrm{OHQ}$ Orthostatic Hypotension Questionnaire.

aFisher's exact test was performed between H\&Y stages and MIBG groups. 


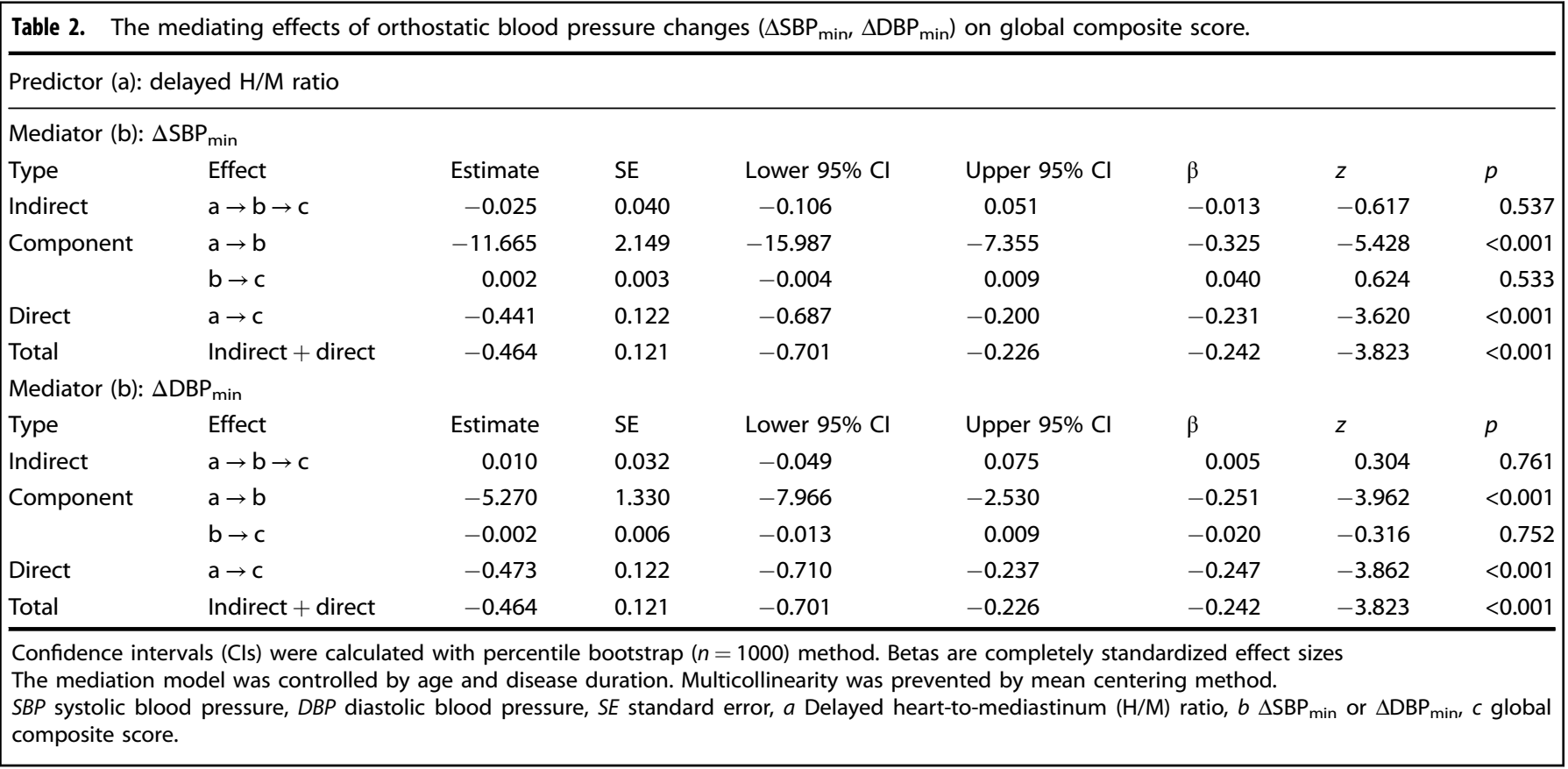

Among the patients studied, $22.9 \%(52 / 227)$ and $26.4 \%(60 / 227)$ were classified into $\mathrm{OH}$ and $\mathrm{OHT}$ groups, respectively.

\section{Comparisons between normal ${ }^{123}$ I-MIBG group and abnormal ${ }^{123}$ I-MIBG group}

The group with low delayed $\mathrm{H} / \mathrm{M}$ ratio had worse outcomes on motor and nonmotor measurements. Total and Part III UPDRS scores were higher (normal ${ }^{123} \mathrm{I}$-MIBG group vs. abnormal ${ }^{123}$ I-MIBG group: total UPDRS, $20.4 \pm 11.1$ vs. $24.8 \pm 12.0$, respectively, $p=0.010$; and for Part III, $13.6 \pm 8.0$ vs. $16.7 \pm 8.7$, respectively, $p=0.013)$. H\&Y stages did not differ between groups (normal ${ }^{123}$ I-MIBG group vs. abnormal ${ }^{123}$ I-MIBG group: 2.0 [1.0] vs. $2.0[0.0], p=0.112$; Fisher's exact test, $p=0.307$ ). The scores of Nonmotor Symptoms Scale (NMSS), Montgomery-Asberg Depression Rating Scale (MADRS), Epworth Sleepiness Scale (ESS), Parkinson's Disease Sleep Scale-2 (PDSS-2), REM Sleep Behavior Disorder Screening Questionnaire (RBDSQ), Scale for Outcomes in Parkinson's Disease-Autonomic (SCOPA-AUT), and the Orthostatic Hypotension Questionnaire (OHQ) Part II were compared between groups; the abnormal ${ }^{123}$ I-MIBG group was scored higher than the normal ${ }^{123}$ I-MIBG group. PD patients with decreased delayed $\mathrm{H} / \mathrm{M}$ ratio had worse overall disease burden than $P D$ patients with normal ${ }^{123}$ I-MIBG (normal ${ }^{123}$ I-MIBG group vs. abnormal ${ }^{123}$ I-MIBG group: global composite score, $-0.3 \pm 0.5$ vs. $0.1 \pm 0.7$, respectively, $p<0.001)$.

Decreased ${ }^{123} \mathrm{I}$-MIBG uptake was associated with $\mathrm{OH}$, while normal ${ }^{123} \mathrm{I}$-MIBG uptake was associated with OHT (Fisher's exact test, $p=0.002, p=0.014$; respectively). The magnitude of blood pressure (BP) drop was higher in the abnormal ${ }^{123}$ I-MIBG group (normal ${ }^{123} \mathrm{I}$-MIBG vs. abnormal ${ }^{123} \mathrm{I}-\mathrm{MIBG}$ : $\triangle \mathrm{SBP}_{\min }, 5.3 \pm 11.0$ vs. $12.9 \pm 13.6$, respectively, $p<0.001 ; \Delta \mathrm{DBP}_{\min }, 0.4 \pm 6.8$ vs. $3.8 \pm 8.0$, respectively, $p=0.002$ ), and $\mathrm{BP}$ rise was greater in the normal ${ }^{123} \mathrm{I}-\mathrm{MIBG}$ group (normal ${ }^{123} \mathrm{I}-\mathrm{MIBG}$ vs. abnormal ${ }^{123} \mathrm{I}-\mathrm{MIBG}$ : $\triangle \mathrm{SBP}_{\max },-3.3 \pm 11.8$ vs. $2.7 \pm 13.8$, respectively, $p=0.002$; $\triangle \mathrm{DBP}_{\max },-5.9 \pm 6.9$ vs. $-3.5 \pm 7.8$, respectively, $p=0.030$ ).

\section{The mediating effects of orthostatic blood pressure changes on clinical outcomes}

Table 2 and Fig. 1 present the mediating effects of BP changes $\left(\triangle \mathrm{SBP}_{\min }, \triangle \mathrm{DBP}_{\min }\right)$ on global composite score. When controlling for age and disease duration, delayed $\mathrm{H} / \mathrm{M}$ ratio was negatively associated with global composite score (total effect [indirect + direct]: $\mathrm{Cl},[-0.701,-0.226])$. Delayed $\mathrm{H} / \mathrm{M}$ ratio was also negatively associated with orthostatic $\Delta \mathrm{BP}_{\min }$ (component $\boldsymbol{a} \rightarrow \boldsymbol{b}$ : $\triangle \mathrm{SBP}_{\min }$ vs. $\triangle \mathrm{DBP}_{\text {min }} ; \mathrm{Cl},[-15.987,-7.355]$ vs. $\mathrm{Cl},[-7.966$, $-2.530]$ ), but its inverse association was not maintained (component $\boldsymbol{b} \rightarrow \boldsymbol{c}: \Delta \mathrm{SBP}_{\min } \mathrm{Vs} . \Delta \mathrm{DBP}_{\min } ; \mathrm{Cl},[-0.004,0.009] \mathrm{vs} . \mathrm{Cl},[-0.013$, $0.009])$. The overall indirect influence was not significant $(\boldsymbol{a} \rightarrow \boldsymbol{b} \rightarrow$ c: $\triangle \mathrm{SBP}_{\min }$ vs. $\triangle \mathrm{DBP}_{\min } ; \mathrm{Cl},[-0.106,0.051]$ vs. $\left.\mathrm{Cl},[-0.049,0.075]\right)$, but the direct negative associations between delayed $\mathrm{H} / \mathrm{M}$ ratio and global composite score were maintained (component $\boldsymbol{a} \rightarrow \boldsymbol{c}$ : $\triangle \mathrm{SBP}_{\text {min }}$ vs. $\triangle \mathrm{DBP}_{\text {min }} ; \mathrm{Cl},[-0.687,-0.200]$ vs. $\left.\mathrm{Cl},[-0.710,-0.237]\right)$. The results were similar when early $\mathrm{H} / \mathrm{M}$ ratio $\left(\boldsymbol{a}^{\prime}\right)$ and washout rate $\left(\boldsymbol{a}^{\prime \prime}\right)$ were set as predictors in the mediation analyses (Supplementary Table 1, Supplementary Fig. 1).

The subdomains of global composite score, partitioned by motor, sleep, and autonomic composite scores were analyzed (Table 3 ). A delayed $\mathrm{H} / \mathrm{M}$ ratio negatively predicted each domain (total effect), and its inverse influence was not mediated by orthostatic $\triangle \mathrm{BP}$ (indirect effect).

\section{Bidirectionality of orthostatic blood pressure change}

In an observational sub-analysis of orthostatic $\Delta \mathrm{BP}_{\max }$ OHT group displayed a worsening trend for global composite score as the $\triangle \mathrm{BP}_{\max }$ decreased while the non-OHT group demonstrated a positive association as the $\Delta B P_{\text {max }}$ increased, especially $\triangle S B P_{\max }$ (Supplementary Fig. 2). These associations lacked any statistical significance; however, orthostatic BP rise and drop was accompanied by trends toward adverse disease severity in both directions.

\section{Comparison of washout rate between OHT and non-OHT}

The washout rate (WR) was significantly opposite (normal ${ }^{123}$ I-MIBG vs. abnormal ${ }^{123}$ I-MIBG: WR, $-4.87 \pm 6.81$ vs. $5.25 \pm$ 7.03 , respectively, $p<0.001)$. In a sub-analysis of sixty-nine normal ${ }^{123}$ I-MIBG patients, $37.7 \%$ (26/69) were orthostatic hypertensive. The WR was compared between those with OHT and without OHT. The comparison did not reveal any significant difference (non-OHT vs. OHT: $-4.39 \pm 7.29$ vs. $-5.65 \pm 5.99$, respectively; independent $t$-test, $t=0.743, d f=67.0, p=0.460)$. 

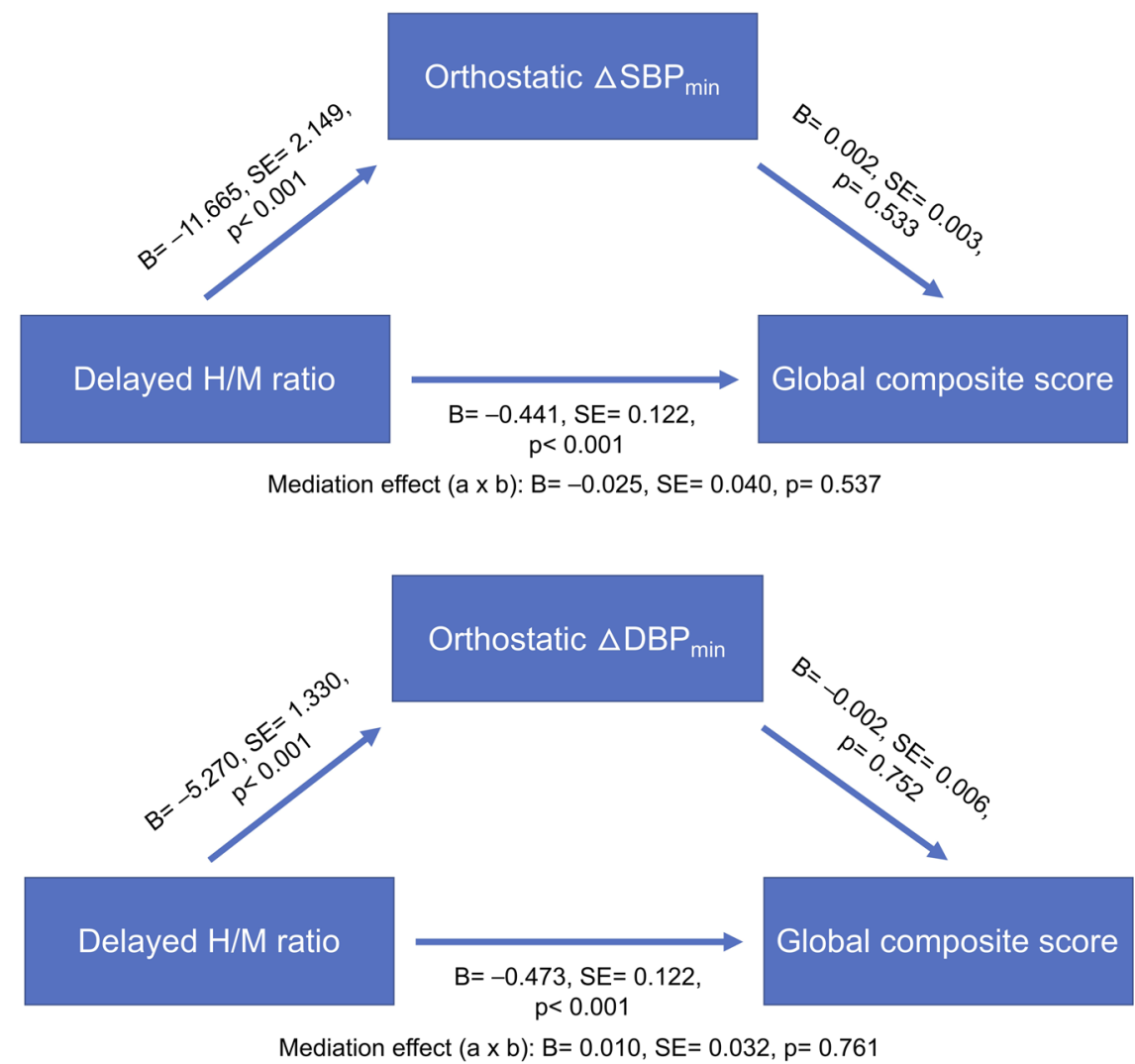

Fig. 1 Path diagram of mediation analysis for global composite score. $H / M$ heart-to-mediastinum, SBP systolic blood pressure, $D B P$ diastolic blood pressure, $B$ estimate, SE standard error.

\section{DISCUSSION}

Cardiac sympathetic denervation and orthostatic hypotension were encountered in early PD patients. $\mathrm{OH}$ was found more frequently in the abnormal ${ }^{123}$-MIBG group while orthostatic hypertension was more common in SWEND PD patients. PD patients with reduced ${ }^{123}$ I-MIBG uptake presented with worse clinical features. Cardiac denervation inversely predicted PDrelated disease burden, irrespective of orthostatic blood pressure changes.

The population of this cohort was in the early mild PD disease stage. The prevalence of abnormal ${ }^{123}$ I-MIBG uptake was comparable to previous studies $7,12,20$. The frequency of $\mathrm{OH}$ in early PD patients was similar to another study ${ }^{21}$, and its association with impaired cardiac sympathetic integrity has been reported ${ }^{4,22}$. In this cohort, PD patients with decreased ${ }^{123}$ I-MIBG uptake showed more severe clinical presentations, and they were more susceptible to orthostatic BP drop ${ }^{7,23}$. PD patients with SWEND were associated with orthostatic BP rise, but had less disease burden.

Irrespective of age and disease duration, more severe cardiac sympathetic denervation was related to a larger drop in orthostatic BP (indirect component $\boldsymbol{a} \rightarrow \boldsymbol{b}$ ) and worse disease severity (direct path $\boldsymbol{a} \rightarrow \boldsymbol{c}$ ). Its negative influence was not carried through BP instability to affect PD patients (indirect path $\boldsymbol{a} \rightarrow \boldsymbol{b} \rightarrow$ c). Thus, the total effect of sympathetic denervation negatively reflected the global disease burden, and was unaffected by BP lability; more severely impaired cardiac dysautonomia was directly associated with worse clinical outcomes (Table 2). Cardiac denervation inversely paralleled motor, sleep, and autonomic global severity domains in the subcomponent analyses (Table 3 ).

The inverse association between cardiac sympathetic denervation and worse disease severity could be explained by the degree of accumulated pathology. In a cross-sectional study, PD patients with cardiac sympathetic denervation were speculated to bear more pathologic burden that resulted in lack of compensatory reserve compared to patients with less cardiac sympathetic denervation, thus worse clinical manifestations ${ }^{7}$. This phenotype was further researched in a longitudinal study, and these patients were shown to be at a higher risk of developing motor complications $^{12}$. This temporal association between PD with abnormal ${ }^{123}$ I-MIBG uptake and wearing-off phenomenon implied that pathologic burden at the periphery could mirror central pathophysiologic disease progression as cardiac sympathetic denervation progressed over time $\mathrm{e}^{2,4,10,12}$. This centripetal degeneration of cardiac sympathetic response was suggested to represent common degenerative process in PD patients ${ }^{1}$, and suggests multiple origination sites for Lewy pathology spread ${ }^{24}$.

The lack of contribution of blood pressure to clinical burdens amid the paths could suggest myocardial ${ }^{123}$ I-MIBG scintigraphy is a potential biomarker that reflects Lewy body pathology ${ }^{2,17}$, in parallel with disease severity. The significant negative association (indirect component $\boldsymbol{a} \rightarrow \boldsymbol{b}$ ), controlled by age and disease duration, confirmed the contributing action of cardiac sympathetic denervation on $\mathrm{OH}^{4}$.

Braak's schema was disputed because its model failed to explain all the subtypes of PD, especially cardiac denervation in early PD ${ }^{24}$. Recent hypothesis of "body-first" subtype PD incorporates the cardiac pathobiology which may be the answer to the observed differences between normal and abnormal ${ }^{123}$ I-MIBG groups in this study ${ }^{25,26}$. This further enhances the extracranial biomarker role of cardiac denervation in PD.

Early $\mathrm{H} / \mathrm{M}$ ratio and washout rate which reflect density of the presynaptic cardiac sympathetic nerve endings ${ }^{2}$, its tone ${ }^{27}$, and damaged or failing myocardium ${ }^{28}$, respectively, also manifested similar results that reinforced the hypothesis that ${ }^{123}$ I-MIBG 


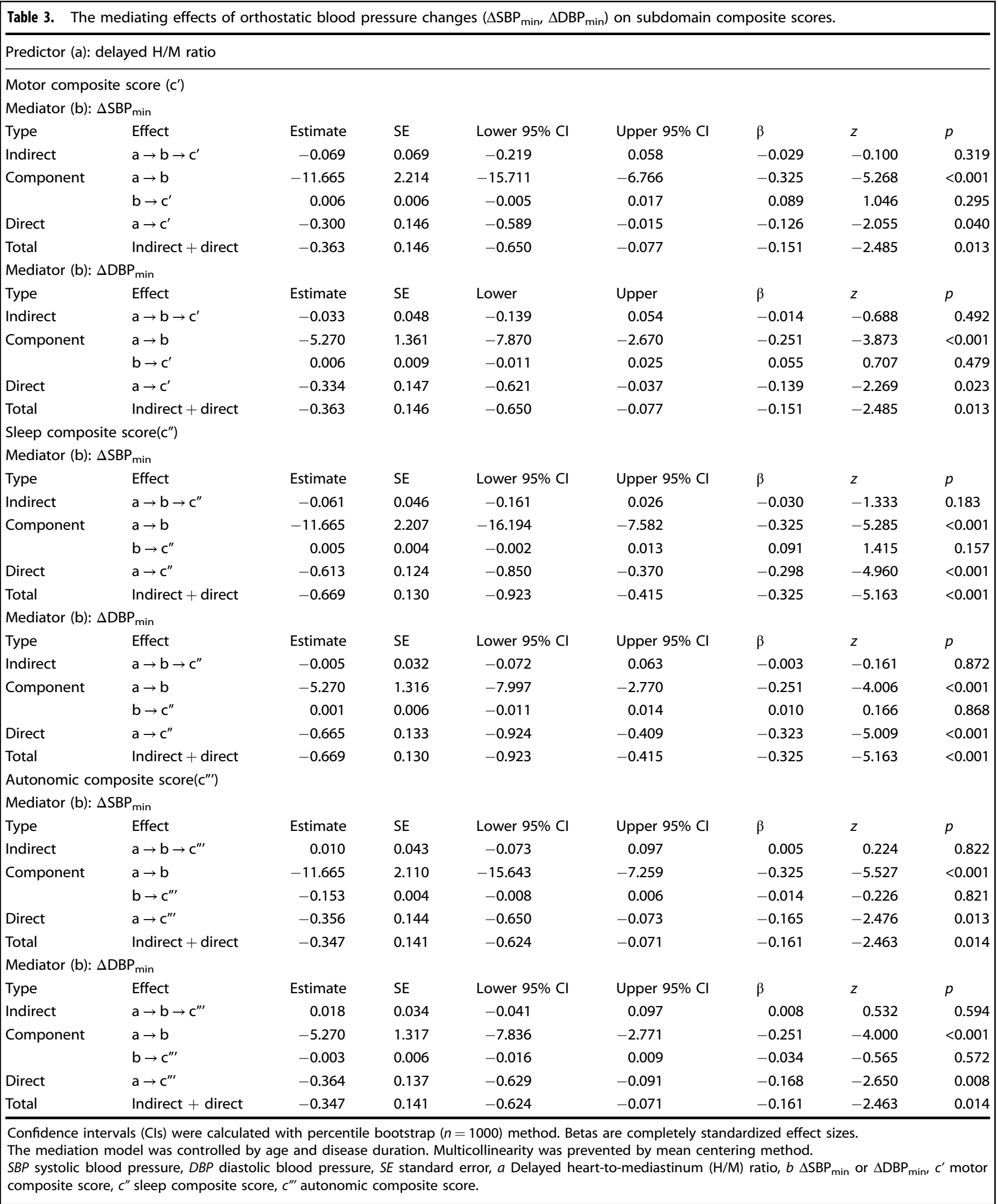

scintigraphy is a mirror of disease burden (Supplementary Table 1, Supplementary Fig. 1).

$\mathrm{OHT}$ has seldom been investigated in PD patients, and its characteristics are largely unknown. In this study, despite a difference in estimation of $\triangle \mathrm{BP}$ during head tilt, the prevalence of $\mathrm{OH}$ and $\mathrm{OHT}$ were comparable. Interestingly, although the analysis did not gain statistical significance, orthostatic BP incremental change was suggested to be related to worse clinical outcomes. 
Orthostatic BP drop also displayed a trend for worse outcomes, particularly with respect to $\triangle \mathrm{SBP}$. OHT is associated with other types of blood pressure lability, and with cardiovascular risk ${ }^{18}$. In accord with our observation, disrupted blood pressure control could be deleterious, regardless of the directionality of the BP changes $^{19}$.

OHT was significantly associated with normal ${ }^{123}$ I-MIBG that included higher values of delayed $\mathrm{H} / \mathrm{M}$ ratios. It also displayed lower and negative washout rate, which apparently signified that delayed $\mathrm{H} / \mathrm{M}$ ratio was greater than early $\mathrm{H} / \mathrm{M}$ ratio. In this regard, delayed $H / M$ and WR could denote the same as WR contains the other in its equation. This was in correspondence to other studies indicating that delayed $\mathrm{H} / \mathrm{M}$ and washout rate represented sympathetic tone ${ }^{2,27}$. Its greater sympathetic tone might have allowed normal ${ }^{123}$ I-MIBG group to culminate into higher orthostatic BP rise, particularly SBP, than abnormal ${ }^{123}$ I-MIBG group.

This result suggested that $\mathrm{OHT}$ could originate by a different cardiovascular dysregulation mechanism since it was related to normal ${ }^{123} \mathrm{I}$-MIBG. As OH in PD stems from cardiac, extra-cardiac denervation and arterial baroreflex failure ${ }^{4}$, orthostatic $\triangle B P$ rise could be the product of compensation of extant cardiac sympathetic tone amid other dysfunctions, which, when lost, could lead to $\mathrm{OH}$. This may imply that $\mathrm{OHT}$ could be a prelude to $\mathrm{OH}$ in some PD. Our data did not depict any significant difference of washout rate between non-OHT and OHT among preserved ${ }^{123}$ I-MIBG group. Had this been manifested, the sick-but-not-dead phenomenon could have played a role in its pathophysiology; ${ }^{29}$ thus, further support the sequential conversion of $\mathrm{OHT}$ into $\mathrm{OH}$ as the disease progresses. The nature of OHT in PD needs to be further assessed.

There are several limitations in this study. First, because the enrolled PD patients were in the early phase of the disease, we could not completely exclude the possibility that some had atypical Parkinsonism. In particular, the PD with SWEND patient group might have inadvertently included some multiple system atrophy patients ${ }^{2}$. We attempted to reduce selection bias by using strict clinical diagnostic criteria for $\mathrm{PD}^{30,31}$ and structural neuroimaging. Nevertheless, it can be difficult to differentiate PD from multiple system atrophy from a nosological perspective. Second, we also could not exclude patients who had genetic Parkinsonism. Patients with the Parkin mutation might not present with nonmotor manifestations and abnormal ${ }^{123}$ I-MIBG uptake $^{32,33}$. This study did not include familial PD or patients with young-onset PD ( $\leq 40$ years). Third, direct biomarkers that provide objective measurements to assess PD disease severity are not available at the present; in addition, currently useful biomarkers do not always represent clinical symptoms. A clinical assessment-based approach, as in this study, is an alternative that evaluates disease severity, relevant to clinical practice. This study incorporated validated clinical tools that encompassed comprehensive motor and nonmotor disease domains. Fourth, there was not sufficient evidence that ${ }^{123}$ I-MIBG uptake represents myocardial synuclein deposition. Future studies that correlate ${ }^{123} \mathrm{I}-\mathrm{MIBG}$ myocardial scans with a direct synuclein accumulation biomarker are warranted. Moreover, cardiac imaging with ${ }^{18} \mathrm{~F}$-fluorodopa, which may be a better substance to evaluate cardiac sympathetic innervation, was also investigated in $\mathrm{PD}^{16,34}$. Diverse studies with cross-validation between different imaging modalities will strongly enhance the results of this research. Finally, because early patients with mild PD stage were enrolled, the scores of questionnaires tended to center at the left of the scale with relatively large standard deviation. A larger recruitment of PD by engagement of multi-clinics to reduce the bias is warranted.

In this cohort, cardiac sympathetic denervation negatively predicted disease severity, independently of orthostatic blood pressure changes. Even though cardiac denervation is a contributor to orthostatic hypotension, the effect of cardiac denervation on disease burdens was not mediated by orthostatic blood pressure changes. Cardiac sympathetic denervation was suggested to represent the disease burden, in parallel with pathologic severity. Orthostatic hypertension was also observed, and it could potentially contribute to PD symptom clinical severity.

\section{METHODS}

\section{Patients}

This study was approved by the Institutional Review Board of Seoul St. Mary's Hospital, and all subjects provided written informed consent to participate. All experiments were conducted in accordance with relevant guidelines and regulations. The study was registered (Identification Number: KCT0005552) in the Clinical Research Information Service (CRIS; http://cris.nih.go.kr), which is an online clinical trial registration system established by the Korea Centers for Disease Control and Prevention (KCDC) with support from the Korea Ministry of Health and Welfare $(\mathrm{KMOHW})$ and is affiliated with the Primary Registries in the World Health Organization (WHO) Registry Network.

Two hundreds twenty-seven de novo and drug-naïve PD patients between April 2014 and January 2020 were enrolled. PD was diagnosed based on the UK Parkinson's Disease Society Brain Bank ${ }^{30}$, and its diagnosis was supported by positron emission tomography imaging studies using ${ }^{18} \mathrm{~F}-\mathrm{N}$-(3-fluoropropyl)-2beta-carbon ethoxy-3beta-(4-iodophenyl) nortropane ${ }^{31}$. All patients had decreased dopamine transporter uptake in the striatum, mainly in the posterior putamen. Patients underwent brain magnetic resonance imaging (MRI) to exclude secondary causes for this finding.

Demographics such as age, sex, body mass index, disease duration, smoking status and history of hypertension, diabetes mellitus, and dyslipidemia were investigated. Disease severity was investigated with the UPDRS and H\&Y stage. Global cognition was assessed by the MMSE and CDR.

Patients were disqualified from the study if they had any of the following indications: (1) any symptoms or signs of atypical and/or secondary Parkinsonism, (2) positive family history of Parkinsonism by pedigree analysis, which included first degree relatives, (3) documentation of atrial fibrillation during the head up tilt test, (4) history of diabetic neuropathy, (5) history of symptomatic stroke that could affect general cognition and performance, and (6) history of medications such as tricyclic antidepressants or benzodiazepines that influence autonomic functions or patients who were taking medications at the time of diagnosis known to influence the central dopaminergic, noradrenergic, and/or serotonergic systems.

Patients were followed every 2-6 months for a minimum of 12 months from the time they began taking dopaminergic medication, and their diagnosis was reaffirmed after at least 12 months of follow-up by two neurologists (S.-W.Y., J.-S.K.).

\section{Questionnaires}

The patients were evaluated with the following questionnaires: (1) NMSS ${ }^{35}$, (2) $\mathrm{MADRS}^{36}$, (3) $\mathrm{ESS}^{37}$, (4) PDSS-2 ${ }^{38}$, (5) RBDSQ ${ }^{39}$, (6) $\mathrm{OHQ}^{40}$, and (7) the SCOPA-AUT ${ }^{41}$. Part I and II of the OHQ were summed separately. Because the SCOPA-AUT sexual dysfunction subsection had too many missing values, this subsection was omitted from that questionnaire's summation. Missing values of sexual subsection was due to patients' reluctance to reveal their sexual activities to the examiner. The sums of each questionnaire were analyzed.

\section{Composite scores}

Motor severity (motor composite score) was gauged by averaging the $z$-scores of the scaled UPDRS II and UPDRS III. The sums of each individual questionnaire for nonmotor features were standardized to z-scores. Nonmotor features were also divided into the sleep domain (sleep composite score: average $z$-scores for the ESS, PDSS-2, RBDSQ), and the autonomic domain (autonomic composite score: average z-scores for the $\mathrm{OHQ}$ and SCOPA-AUT), and were further analyzed separately. The affection domain was not analyzed because the MADRS data were not normally distributed, and the NMSS was excluded from subcomponent analysis due to its inclusive psychometric properties.

Parkinson's disease overall burden (the global composite score) was estimated by averaging the scaled z-scores of all motor and nonmotor 
assessments. Higher z-scores indicated worse severity. All questionnaires were evaluated by investigators blind to patient clinical information.

\section{Head-up tilt test}

All patients were tested in the full resting state. Continuous electrocardiograph leads and non-invasive BP monitoring equipment were applied to the patients (YM6000, Mediana Tech, Redmond, WA, USA). A supine position was maintained for 20 min during recording of BPs and heart rates every $5 \mathrm{~min}$, before tilting to 60 degrees (ENRAF NONIUS, Rotterdam, The Netherlands). At the tilted position, the same measurements were taken at $0,3,5,10,15$, and $20 \mathrm{~min}$.

After excluding the first supine BP at 0 min, average supine systolic and diastolic BPs were estimated from the measurements at 5, 10, 15, and $20 \mathrm{~min}$. SH was defined if the average supine systolic and/or diastolic BPs (SBP/DBP) were $\geq 140 / 90 \mathrm{mmHg}^{42}$

The lowest SBP/DBP at 3 or 5 minutes during the tilted position were selected for the diagnosis of $\mathrm{OH}$. The orthostatic BP changes in systole $\left(\triangle \mathrm{SBP}_{\min }\right)$ and diastole $\left(\triangle \mathrm{DBP}_{\min }\right)$ were also calculated (supine average $\mathrm{BP}$ minus lowest orthostatic $B P$ ). When patients were hypertensive with $\geq 140$ / $90 \mathrm{mmHg}$ in the supine position, $\triangle \mathrm{SBP}_{\text {min }}$ and/or $\triangle \mathrm{DBP}_{\min } \geq 30 / 15 \mathrm{mmHg}$ within $5 \mathrm{~min}$ was applied to define $\mathrm{OH}$; otherwise, $\triangle \mathrm{SBP}_{\min }$ and/or $\triangle \mathrm{DBP}_{\min }$ $\geq 20 / 10 \mathrm{mmHg}$ were adopted ${ }^{43}$

The highest SBP/DBP among tilted measurements at 3,5, 10, 15, and 20 min were re-selected, and the orthostatic $\triangle B P s$ were calculated from the average BPs (supine average BP minus highest orthostatic BP). PD patients with $\mathrm{SH}$ were defined as having orthostatic hypertension $(\mathrm{OHT})$ if $\triangle \mathrm{SBP}_{\max }$ and/or $\triangle \mathrm{DBP}_{\max }$ was $\leq-20 / 10 \mathrm{mmHg}$. PD patients without $\mathrm{SH}$ were categorized as $\mathrm{OHT}$ when their orthostatic $\mathrm{BP}_{\max }$ was $\geq 140 / 90 \mathrm{mmHg}$ or $\triangle \mathrm{BP}_{\max }$ was $\leq-20 / 10 \mathrm{mmHg}^{19}$.

Positive orthostatic $\triangle \mathrm{BP}$ indicated a drop in standing $\mathrm{BP}$, and the negative $\triangle B P$ an increase in standing $B P$.

\section{${ }^{123} \mathrm{I}$-metaiodobenzylguanidine ( $\left.{ }^{123} \mathrm{I}-\mathrm{MIBG}\right)$ scintigraphy}

${ }^{123}$ I-MIBG scintigraphy was performed using a dual-head camera equipped with a low-energy, high-resolution collimator (Siemens), and data were collected at 30-min (early) and 2-h (delayed) time points after the injection of $111 \mathrm{MBq}$ of ${ }^{123}$-MIBG. A static image was obtained with a $128 \times 128$ matrix. Regions of interest were manually drawn around the heart and mediastinum. Tracer uptake was measured within each region of interest to calculate the $\mathrm{H} / \mathrm{M}$ ratio. The lower limit of the reference value for delayed $\mathrm{H} / \mathrm{M}$ ratio was calculated to be $1.78^{12,13}$. A delayed $\mathrm{H} / \mathrm{M}$ ratio < 1.78 was defined as abnormal. Washout rate was calculated as the following: [(early $\mathrm{H} / \mathrm{M}$ ratio - late $\mathrm{H} / \mathrm{M}$ ratio)/early $\mathrm{H} / \mathrm{M}$ ratio] $\times 100^{13}$.

\section{Statistical analyses}

All statistical analyses were conducted with Jamovi software (version 1.6. retrieved from https://www.jamovi.org) for the Mac, a graphical user interface for $R$, with the additional jAMM module. The jAMM module provides a GLM mediation model that utilizes the lavaan $\mathrm{R}$ package ${ }^{44}$. Descriptive statistics, and independent or Welch's t-tests, the Mann-Whitney $U$ test or Fisher's exact test were performed as appropriate. Mediation models, partialized by the covariates of age and disease duration, were manipulated to assess whether there was a mediating role for orthostatic $\triangle \mathrm{BP}_{\min }(\boldsymbol{b})$ between cardiac sympathetic denervation $(\boldsymbol{a})$ and disease burden (c). The direct association between delayed $\mathrm{H} / \mathrm{M}$ ratio and composite scores $(\boldsymbol{a} \rightarrow \boldsymbol{c})$ was established within the context of an indirect effect of orthostatic $\Delta \mathrm{BP}_{\min }(\boldsymbol{a} \rightarrow \boldsymbol{b} \rightarrow \boldsymbol{c})$. Their relative roles were estimated using the maximum likelihood method ${ }^{45}$ and statistical significance was defined as a two-tailed $p$ value $<0.05$.

\section{Reporting summary}

Further information on research design is available in the Nature Research Reporting Summary linked to this article.

\section{DATA AVAILABILITY}

Anonymized data generated during the current study are available from the corresponding author on reasonable request from individuals affiliated with research or health care institutions.

\section{CODE AVAILABILITY}

Jamovi is a statistical spreadsheet and graphical user interface (GUI) for R. All the analyses were performed basically using jamovi software as mentioned in the method, statistical analysis section. The $\mathrm{R}$ package mentioned was utilized within jamovi GUI.

Received: 11 March 2021; Accepted: 23 July 2021; Published online: 12 August 2021

\section{REFERENCES}

1. Orimo, S. et al. Axonal alpha-synuclein aggregates herald centripetal degeneration of cardiac sympathetic nerve in Parkinson's disease. Brain 131, 642-650 (2008).

2. Orimo, S., Yogo, M., Nakamura, T., Suzuki, M. \& Watanabe, H. (123)I-meta-iodobenzylguanidine (MIBG) cardiac scintigraphy in a-synucleinopathies. Ageing Res. Rev. 30, 122-133 (2016).

3. Goldstein, D. S. Orthostatic hypotension as an early finding in Parkinson's disease. Clin. Auton. Res. 16, 46-54 (2006).

4. Jain, S. \& Goldstein, D. S. Cardiovascular dysautonomia in Parkinson disease: from pathophysiology to pathogenesis. Neurobiol. Dis. 46, 572-580 (2012).

5. Schrag, A., Horsfall, L., Walters, K., Noyce, A. \& Petersen, I. Prediagnostic presentations of Parkinson's disease in primary care: a case-control study. Lancet Neurol. 14, 57-64 (2015).

6. Choi, M. H., Yoon, J. H. \& Yong, S. W. Cardiac sympathetic denervation and dementia in de novo Parkinson's disease: a 7-year follow-up study. J. Neurol. Sci. 381, 291-295 (2017).

7. Kim, J. S. et al. Normal 'heart' in Parkinson's disease: is this a distinct clinical phenotype? Eur. J. Neurol. 24, 349-356 (2017).

8. Kotagal, V., Lineback, C., Bohnen, N. I. \& Albin, R. L. Orthostatic hypotension predicts motor decline in early Parkinson disease. Parkinsonism Relat. Disord. 32, 127-129 (2016).

9. Fereshtehnejad, S. M. et al. New clinical subtypes of Parkinson disease and their longitudinal progression: a prospective cohort comparison with other phenotypes. Jama. Neurol. 72, 863-873 (2015).

10. Tsujikawa, K. et al. Chronological changes of 123I-MIBG myocardial scintigraphy and clinical features of Parkinson's disease. J. Neurol. Neurosurg. Psychiatry $\mathbf{8 6}$ 945-951 (2015).

11. Sakakibara, R. et al. MIBG myocardial scintigraphy in pre-motor Parkinson's disease: a review. Parkinsonism Relat. Disord. 20, 267-273 (2014).

12. Lee, J. E. et al. Cardiac sympathetic denervation can predict the wearing-off phenomenon in patients with Parkinson disease. J. Nucl. Med. 59, 1728-1733 (2018).

13. Ryu, D. W. et al. Initial versus follow-up sequential myocardial 123I-MIBG scintigraphy to discriminate Parkinson disease from atypical Parkinsonian syndromes. Clin. Nucl. Med. 44, 282-288 (2019).

14. Hamada, K. et al. Onset age and severity of motor impairment are associated with reduction of myocardial 123I-MIBG uptake in Parkinson's disease. J. Neurol. Neurosurg. Psychiatry 74, 423-426 (2003).

15. Spiegel, J. et al. Myocardial sympathetic degeneration correlates with clinical phenotype of Parkinson's disease. Mov. Disord. 22, 1004-1008 (2007).

16. Goldstein, D. S. \& Cheshire, W. P. Jr. Roles of cardiac sympathetic neuroimaging in autonomic medicine. Clin. Auton. Res. 28, 397-410 (2018).

17. Spiegel, J. Diagnostic and pathophysiological impact of myocardial MIBG scintigraphy in Parkinson's disease. Parkinsons Dis. 2010, 295346 (2010).

18. Robertson, D. The pathophysiology and diagnosis of orthostatic hypotension. Clin. Auton. Res. 18, S2-S7 (2008).

19. Jordan, J., Ricci, F., Hoffmann, F., Hamrefors, V. \& Fedorowski, A. Orthostatic hypertension: critical appraisal of an overlooked condition. Hypertension 75, 1151-1158 (2020).

20. Kawazoe, M. et al. Sensitivity and specificity of cardiac (123)I-MIBG scintigraphy for diagnosis of early-phase Parkinson's disease. J. Neurol. Sci. 407, 116409 (2019).

21. Hiorth, Y. H., Pedersen, K. F., Dalen, I., Tysnes, O. B. \& Alves, G. Orthostatic hypotension in Parkinson disease: a 7-year prospective population-based study. Neurology 93, e1526-e1534 (2019).

22. Nakamura, T. et al. Role of cardiac sympathetic nerves in preventing orthostatic hypotension in Parkinson's disease. Parkinsonism Relat. Disord. 20, 409-414 (2014).

23. Kim, J. S. et al. Orthostatic hypotension and cardiac sympathetic denervation in Parkinson disease patients with REM sleep behavioral disorder. J. Neurol. Sci. 362, 59-63 (2016)

24. Rietdijk, C. D., Perez-Pardo, P., Garssen, J., van Wezel, R. J. \& Kraneveld, A. D. Exploring Braak's hypothesis of Parkinson's disease. Front. Neurol. 8, 37 (2017).

25. Borghammer, P. The a-synuclein origin and connectome model (SOC Model) of Parkinson's disease: explaining motor asymmetry, non-motor phenotypes, and cognitive decline. J. Parkinsons Dis. 11, 455-474 (2021). 
26. Horsager, J. et al. Brain-first versus body-first Parkinson's disease: a multimodal imaging case-control study. Brain 143, 3077-3088 (2020).

27. Jeong, Y. J. et al. Relationship between the washout rate of I-123 MIBG scans and autonomic function in Parkinson's disease. PLoS. One 15, e0229860 (2020).

28. Nakajima, K., Taki, J., Tonami, N. \& Hisada, K. Decreased 123I-MIBG uptake and increased clearance in various cardiac diseases. Nucl. Med. Commun. 15, 317-323 (1994).

29. Goldstein, D. S. The "Sick-but-not-Dead" phenomenon applied to catecholamine deficiency in neurodegenerative diseases. Semin Neurol. 40, 502-514 (2020).

30. Gibb, W. R. \& Lees, A. J. The relevance of the Lewy body to the pathogenesis of idiopathic Parkinson's disease. J. Neurol. Neurosurg. Psychiatry 51, 745-752 (1988).

31. Postuma, R. B. et al. MDS clinical diagnostic criteria for Parkinson's disease. Mov. Disord. 30, 1591-1601 (2015).

32. Kim, Y. D. et al. Cardiac (123)l-metaiodobenzylguanidine scintigraphy in a patient with familial parkinsonism with Parkin gene mutation. J. Mov. Disord. 3, 42-44 (2010).

33. Kägi, G. et al. Nonmotor symptoms in Parkin gene-related parkinsonism. Mov. Disord. 25, 1279-1284 (2010).

34. Goldstein, D. S., Sewell, L. \& Sharabi, Y. Autonomic dysfunction in PD: a window to early detection? J. Neurol. Sci. 310, 118-122 (2011).

35. Koh, S. B. et al. Validation of the Korean-version of the nonmotor symptoms scale for Parkinson's disease. J. Clin. Neurol. 8, 276-283 (2012).

36. Ahn, Y. M. et al. A validation study of the Korean-version of the Montgomery-Asberg depression rating scale. J. Korean Neuropsychiatr. Assoc. 44, 466-476 (2005).

37. Cho, Y. W. et al. The reliability and validity of the Korean version of the Epworth sleepiness scale. Sleep. Breath. 15, 377-384 (2011).

38. Yang, H. J. et al. Subtypes of sleep disturbance in Parkinson's disease based on the cross-culturally validated Korean Version of Parkinson's Disease Sleep Scale-2. J. Clin. Neurol. 16, 66-74 (2020).

39. Lee, S. A., Paek, J. H., Han, S. H. \& Ryu, H. U. The utility of a Korean version of the REM sleep behavior disorder screening questionnaire in patients with obstructive sleep apnea. J. Neurol. Sci. 358, 328-332 (2015).

40. Kaufmann, H., Malamut, R., Norcliffe-Kaufmann, L., Rosa, K. \& Freeman, R. The Orthostatic Hypotension Questionnaire (OHQ): validation of a novel symptom assessment scale. Clin. Auton. Res. 22, 79-90 (2012).

41. Kim, J. Y. et al. Validation of the Korean version of the Scale for Outcomes in Parkinson's Disease-Autonomic. J. Mov. Disord. 10, 29-34 (2017).

42. Fanciulli, A. et al. Consensus statement on the definition of neurogenic supine hypertension in cardiovascular autonomic failure by the American Autonomic Society (AAS) and the European Federation of Autonomic Societies (EFAS): Endorsed by the European Academy of Neurology (EAN) and the European Society of Hypertension (ESH). Clin. Auton. Res. 28, 355-362 (2018).

43. Gibbons, C. H. et al. The recommendations of a consensus panel for the screening, diagnosis, and treatment of neurogenic orthostatic hypotension and associated supine hypertension. J. Neurol. 264, 1567-1582 (2017).

44. Rosseel, Y. lavaan: An R package for structural equation modeling. J. Stat. Soft $\mathbf{4 8 ,}$ 1-36 (2012)

45. Rijnhart, J. J. M., Twisk, J. W. R., Chinapaw, M. J. M., de Boer, M. R. \& Heymans, M. W. Comparison of methods for the analysis of relatively simple mediation models. Contemp. Clin. Trials Commun. 7, 130-135 (2017).

\section{ACKNOWLEDGEMENTS}

This research was supported by Basic Science Research Program through the National Research Foundation of Korea (NRF) funded by the Ministry of Science, ICT and Future Planning (NRF-2017R1D1A1B06028086). This study was also supported by Research Fund of Seoul St. Mary's Hospital, The Catholic University of Korea.

\section{AUTHOR CONTRIBUTIONS}

S-.W.Y., and J-S.K. contributed the conception and design of the study; S-.W.Y., J-S.K., Y-.,S.O., D-.W.R., S.H., J-Y.Y., and K-.S.L. contributed to the acquisition and analysis of data; S-.W.Y., and J-S.K. contributed to the interpretation of results drafting the text and preparing figure; S-.W.Y. drafted the manuscript; J-S.K., Y-.,S.O., D-.W.R., S.H., J-.Y.Y., and K-.S.L. revised the manuscript. S-.W. Y., and J-S.K. obtained funding. All authors read and approved the final version for publication.

\section{COMPETING INTERESTS}

The authors declare no competing interests.

\section{ADDITIONAL INFORMATION}

Supplementary information The online version contains supplementary material available at https://doi.org/10.1038/s41531-021-00217-3.

Correspondence and requests for materials should be addressed to J.-S.K.

Reprints and permission information is available at http://www.nature.com/ reprints

Publisher's note Springer Nature remains neutral with regard to jurisdictional claims in published maps and institutional affiliations.

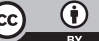

Open Access This article is licensed under a Creative Commons Attribution 4.0 International License, which permits use, sharing, adaptation, distribution and reproduction in any medium or format, as long as you give appropriate credit to the original author(s) and the source, provide a link to the Creative Commons license, and indicate if changes were made. The images or other third party material in this article are included in the article's Creative Commons license, unless indicated otherwise in a credit line to the material. If material is not included in the article's Creative Commons license and your intended use is not permitted by statutory regulation or exceeds the permitted use, you will need to obtain permission directly from the copyright holder. To view a copy of this license, visit http://creativecommons. org/licenses/by/4.0/.

(c) The Author(s) 2021 